\title{
Consumption of foods by young children with diagnosed campylobacter infection - a pilot case-control study
}

\author{
Scott Cameron 1,2, Karin Ried ${ }^{2}$, Anthony Worsley ${ }^{2,3}$ and David Topping ${ }^{4, *}$ \\ ${ }^{1}$ Communicable Disease Control Branch, Department of Human Services, South Australian Government, Adelaide, \\ Australia: ${ }^{2}$ Department of Public Health, The University of Adelaide, Adelaide, Australia: ${ }^{3}$ School of Health Sciences, \\ Deakin University, Burwood, Australia: ${ }^{4}$ CSIRO Health Sciences and Nutrition, Kintore Avenue, PO Box 10041, \\ Adelaide, South Australia 5000, Australia
}

Submitted 20 March 2003: Accepted 22 July 2003

\begin{abstract}
Objective: To determine whether parentally reported habitual intake of specific foods differed between children with diagnosed Campylobacter jejuni infection and children of a comparison group without diagnosed infection.

Design, setting and subjects: Information was collected from the parents or primary caregivers of South Australian children aged 1-5 years with diagnosed $C$. jejuni (cases, $n=172$ ) and an age- and gender-matched group of uninfected children (controls, $n=173$ ). Frequency of consumption of 106 food and drink items was determined for the preceding two months by food-frequency questionnaire. Four children in the control group had recorded diarrhoeal episodes during the assessment period and were excluded, so 169 responses were evaluated for this group. Information was gathered on possible confounders including socio-economic status. Response frequencies were classified into three levels of consumption (rarely, weekly or daily) and statistical comparison was made by frequency of consumption of foods versus the 'rarely' classification for cases and controls, respectively.

Results: Frequency of consumption of most foods, including starchy foods and fruits and vegetables, did not differ between cases and controls. However, reported consumption of eight food items (block and processed cheese (slices and spread), salami/fritz (a form of processed sausage), chicken nuggets, pasteurised milk, fish (canned or fresh) and hot French fries) was significantly higher by controls.

Conclusions: The hypothesis that reported consumption of starchy foods was lower by cases than by controls was not supported by the data. However, consumption of some processed and unprocessed foods was higher by controls. Some of these foods have established bactericidal actions in vitro that may indicate a possible mechanism for this apparent protection.
\end{abstract}

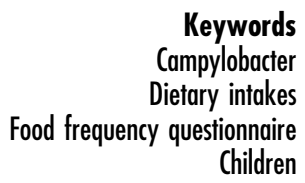

Keywords

Campylobacter

estionnaire

Children
The relationships between poor sanitation, food and domestic water quality and increased risk of infectious diarrhoeal diseases are well established ${ }^{1}$. While morbidity and mortality from these causes is much lower in affluent economies with high standards of personal and food industry hygiene, the reported rates of infection remain surprisingly high $^{2}$. For example, approximately 10000 cases of campylobacter infection are reported annually in Australia (excluding the largest state, New South Wales, where the disease is not notifiable) $)^{3}$. The highest rates for this infection are found in young children ${ }^{3-5}$. In the year 2000, 331 cases in children aged $1-4$ years were reported to the South Australian Communicable Disease Control Branch. This accounted for almost $20 \%$ of total notifications for that year.
Reported risk factors for campylobacter infection include ownership of domestic pets (especially puppies and kittens), consumption of raw (i.e. unpasteurised) milk and undercooked chicken meat, exposure to untreated water and poor food hygiene ${ }^{3}$. Current methods of public health investigation of cases seem to focus on the food supply as a potential vector but not for any potential role in altering the susceptibility of individuals to infection. There is an emerging body of evidence for an important interaction between dietary components and the microbial population of the normal human gastrointestinal tract ${ }^{6,7}$. Much of this investigation relates to the large and taxonomically diverse population resident in the large bowel, where over 50 genera and 400 species have been identified $^{6}$. These bacteria are mostly non-pathogenic, 
fermenting undigested dietary components (principally carbohydrates) and endogenous secretions. In adults and children eating solid foods, short-chain fatty acids are substantial end products of this metabolism and contribute to normal colonic function by a number of actions including inhibiting the overgrowth of potentially pathogenic micro-organisms and stimulating fluid and electrolyte absorption ${ }^{7,8}$. Both of the latter effects would be expected to assist in the host defence against diarrhoeal diseases. The numbers and metabolic activities of the large bowel microflora can be modulated favourably through the consumption of specific foods and food ingredients termed prebiotics ${ }^{6}$. Resistant starch (RS) is one such prebiotic $^{8}$, and has been shown to accelerate recovery in children infected by cholera ${ }^{9}$ and to control other infectious diarrhoeal diseases in children ${ }^{10}$ and weaning piglets $^{11}$. Based on this background knowledge, we postulated that its consumption, as part of the habitual diet, could play a role in the aetiology of infectious intestinal disease through altering host susceptibility. Starch (and RS) intakes are low in Australia and other Westernised industrial countries ${ }^{12,13}$, which should contribute to greater host susceptibility to diarrhoeal illness. This hypothesis was examined in a pilot study by assessing patterns of food consumption in young children with and without reported campylobacter infection. Currently, there is no validated assay for dietary RS. However, it is thought to be a function (possibly 10\%) of total starch consumption $^{7,8,12}$, so that dietary starch provides an indirect measure of RS intake. Here we report that, indeed, certain dietary intakes (as assessed by food-frequency questionnaires completed by parents) are higher in young children not affected by this illness. However, these intakes were not of starchy foods in general but of some other processed and convenience foods, including cheese products.

\section{Methods}

\section{Ethical considerations}

The study protocol and questionnaire were approved by the University of Adelaide Human Experimentation Ethics Committee.

\section{Study population and data collection}

Children aged $1-5$ years with Campylobacter jejuni infection notified to the Communicable Disease Control Branch of the South Australian Department of Human Services between January 2000 and January 2001 were defined as cases. The majority (73\%) of the comparison group (controls), who were similar to cases in terms of age and sex distribution, were selected randomly from the metropolitan and rural areas within South Australia using a computer-assisted telephone interview system that has been described previously ${ }^{14}$. The remaining controls were drawn randomly from the South Australian birth registry.
In total, the parents, guardians or principal caregivers of 351 cases and 241 controls were contacted by telephone and/or letter, seeking information on the children's dietary habits. These habits were ascertained by means of a foodfrequency questionnaire modified from those used by Baghurst et al. ${ }^{15}$ and the Australian Bureau of Statistics for the 1995 National Nutrition Survey ${ }^{16}$. The modified questionnaire was developed in concert with Consumer Sciences Programme, CSIRO Health Sciences and Nutrition. Overall, completed questionnaires were received from 172 (66\%) of the cases and 173 (87\%) of the controls who indicated initial agreement to participate.

Questions were asked about the frequency of consumption of a total of 106 food and drink items in the two months prior to completing the questionnaire. Individual sections of the questionnaire were assigned to 'meat and fish', 'cereals and breads', 'drinks', 'fruit and vegetables', 'dairy foods', 'snacks and sweets' and 'spreads and oils'. Response frequencies were split into six groups in the questionnaire and further condensed into three categories for analysis. Food items in category 1 were consumed rarely to twice a month, between one and four times a week in category 2, and daily in category 3. The questionnaire also sought information on possible confounders including the child's recent health (specifically recent gastrointestinal symptoms), the family's health (notably any family history of bowel cancer), history of breast- or bottle-feeding, family smoking habits and socioeconomic status (determined by household income and parental education level). Information was sought also on the keeping of pets or animals. Copies of the questionnaire are available on request from the corresponding author.

\section{Data entry and analysis}

The experimental design was a comparison of the parental or guardian-reported frequency of consumption of foods set against group 1 ('rarely') for children who were cases or controls, respectively. Potential confounding was minimised by ensuring (as far as possible) a similar age and gender distribution in cases and controls. Ageadjusted odds ratios (ORs) and 95\% confidence intervals (CIs) were calculated for each variable using this group as a reference. Individuals were assigned to 1-year age groups by their birthday, so that age group 1 includes children who were $>5$ months and $<1.5$ years old. The age and gender distributions for cases in each group were matched as far as possible. To control for undiagnosed diarrhoeal disease (which could have been due to campylobacter infection), parental response was sought about recent gut illness. Four of the 173 controls were reported to have experienced diarrhoea for more than 6 days in the 2 months prior to completion of the questionnaire. Since we cannot rule out an infective cause for these episodes (e.g. undiagnosed campylobacter infection), their data were excluded from the final analysis. The data presented in this paper are therefore based on 
the parental responses for 172 diagnosed cases and 169 controls.

Data from the completed questionnaires were entered into a computer spreadsheet by use of the Microsoft Access data management program. Statistical analysis was carried out using the Stata 7 program ${ }^{17}$.

\section{Results}

\section{Characteristics of cases and controls}

Of the 172 cases, 96 were male and 76 were female, while there was a slightly different profile in the controls with 91 males and 78 females. Stratification by age group showed that the number of cases rose from age 1-3 years and declined thereafter (data not shown). Cases and controls were comparable with respect to parental income, education and smoking, as well as history of breast-feeding.

\section{Consumption of foods and beverages}

There were no differences in the frequency of consumption of most foods between diagnosed cases and controls. These included a wide range of fruits and vegetables, meats, snacks and sweets, breads and cereals, and spreads and oils. The beverages included reduced-fat milk and tap or bottled water. However, the frequency of consumption of eight food items was significantly higher by controls than by diagnosed cases (Table 1). The foods that showed substantially lower ORs with greater consumption by controls were block cheese (consumed daily or weekly), processed cheese (cheese slices and cheese spread), salami/fritz (a type of pre-cooked sausage), chicken nuggets, pasteurised milk, fish (canned or fresh) and hot French fries, with ORs ranging from 0.33 for cheese slices (consumed daily) to 0.64 for French fries. In contrast, three foods - oat porridge, butter and sweet potatoes - were consumed more frequently by cases than by controls (Table 2).

The keeping of live chickens was found to be higher in cases (OR 4.5, 95\% CI 1.5-14.0, P<0.004), as was a history of colon cancer in the immediate family (OR 1.9, 95\% CI 1.1-3.3, $P<0.01)$.

\section{Discussion}

Campylobacter is an acute, notifiable infection with a range of symptoms including diarrhoea. The condition was selected to study the relationship between parentalreported dietary intakes and environmental infection in young children for several reasons. First, the condition is common, especially in the period after weaning ${ }^{3,18}$. Second, despite reports that certain foods or lifestyle factors may be vectors, the source of infection for most cases is unclear and may be found in the child's general environment (rather than being due to the consumption of specific food sources). An example of this is the outbreak of yersioniosis recorded in Georgia (USA) in 1988-1989.

Table 1 Frequency of consumption of foods and beverages apparently protective against diagnosed campylobacter infection in children

\begin{tabular}{|c|c|c|c|c|c|c|}
\hline Food/drink item & OR & $95 \% \mathrm{Cl}$ & $P$-value & $\begin{array}{c}\text { Consumed by } \\
\text { controls/cases (\%) }\end{array}$ & $\begin{array}{c}\text { Consumed by } \\
\text { controls/cases (\%) }\end{array}$ & $\begin{array}{l}\text { Sample sizeł, } \\
\text { controls/cases }\end{array}$ \\
\hline 1 Cheese slices & $0.33^{*}$ & $0.15-0.71$ & 0.003 & $22.3 / 12.9^{\star}$ & $70.5 / 59.4$ & $166 / 170$ \\
\hline 2 Block cheese & $0.38^{*}$ & $0.19-0.76$ & 0.005 & $25.3 / 16.6^{\star}$ & $83.1 / 71.6$ & $166 / 169$ \\
\hline 3 Salami/fritz† & 0.52 & $0.32-0.83$ & 0.006 & $51.2 / 34.3$ & $54.9 / 39.8$ & $162 / 166$ \\
\hline 4 Chicken nuggets & 0.52 & $0.30-0.91$ & 0.012 & $28.8 / 17.9$ & $29.5 / 17.9$ & $163 / 168$ \\
\hline 5 Cheese spread & 0.55 & $0.32-0.97$ & 0.035 & $25.9 / 16.8$ & $28.4 / 18.6$ & $162 / 167$ \\
\hline 6 Milk (full-cream) & $0.53^{*}$ & $0.29-0.97$ & 0.036 & $73.5 / 61.5^{\star}$ & $85.5 / 78.1$ & $166 / 169$ \\
\hline 7 Fish (canned or fresh) & 0.59 & $0.35-0.98$ & 0.040 & $26.6 / 17.5$ & $26.9 / 17.7$ & $167 / 169$ \\
\hline 8 French fries & 0.64 & $0.40-1.01$ & 0.052 & $62.3 / 49.4$ & $62.3 / 46.8$ & $167 / 170$ \\
\hline
\end{tabular}

OR - age-adjusted odds ratio; $\mathrm{Cl}$ - confidence interval.

* Daily consumption, otherwise weekly consumption.

$\dagger$ Including weekly and daily consumption.

$\ddagger$ Number of subjects who responded to the particular question; overall sample sizes for controls/cases $=169 / 172$.

Table 2 Frequency of consumption of foods apparently increasing the risk of diagnosed campylobacter infection in children

\begin{tabular}{lccccc}
\hline \multicolumn{1}{c}{ Food item } & Odds ratio & $95 \% \mathrm{Cl}$ & $P$-value & $\begin{array}{c}\text { Consumed by } \\
\text { controls/cases (\%) }\end{array}$ & $\begin{array}{c}\text { Sample size } \neq \\
\text { controls/cases }\end{array}$ \\
\hline 1 Porridge & 2.20 & $1.22-3.98$ & 0.007 & $14.5 / 29.0$ & $165 / 169$ \\
2 Butter & $1.91^{*}$ & $1.11-3.30$ & 0.018 & $43.7 / 55.6$ & $158 / 160$ \\
3 Sweet potatoes & 1.64 & $0.97-2.76$ & 0.060 & $18.6 / 29.5$ & $167 / 166$ \\
\hline
\end{tabular}

OR - age-adjusted odds ratio; $\mathrm{Cl}$ - confidence interval.

* Daily consumption, otherwise weekly consumption.

†Including weekly and daily consumption.

$\ddagger$ Number of subjects who responded to the particular question; overall sample sizes for

controls/cases $=169 / 172$. 
In that instance, the organisms were transmitted from contaminated foods to children via the hands of food handlers $^{19}$. Finally, if foods do play a role in host susceptibility in this age group, dietary modification could be a very useful means of public health intervention.

Our initial hypothesis was that there was a relationship between inadequate intakes of RS (measured indirectly through the consumption of starchy foods) and campylobacter infection. This expectation was based on numerous reports of the beneficial effects of nutritional interventions with dietary complex carbohydrates on intestinal microbiology in humans ${ }^{9,10}$ and animals ${ }^{8,11}$. This proposition appears to be unsupported by the current data except for French fries, which are foods high in fat as well as starch (although the RS content of French fries is unknown). The frequency of consumption of breads, cereals, etc. by controls was the same as by cases and the single cereal-based item to approach significance was bread as rolls (OR 0.67, 95\% CI 0.42-1.06, $P<0.084$ ).

The survey included questions for a range of other foods and beverages and revealed that there were significant associations. Prominent among these were higher reported frequencies of consumption of block cheese (cheddar), processed cheese (cheese slices) and cheese spreads by controls. The difference between hypothesis and outcome may reflect the specific pathology of campylobacter infection. It may also reflect a difference between factors altering susceptibility to infection rather than the recovery from disease, which has been the focus of research on RS. There is already supporting evidence that the consumption of some cheese products (curd and cottage cheese) is lower in individuals infected with campylobacter than in controls ${ }^{20}$, with ORs similar to those found in the present study. When cheese is inoculated with pathogens (including campylobacter) their survival times are short ${ }^{21}$, suggesting the presence of bactericidal components. Cheese and other food items that were eaten more often by controls have a relatively high fat content. In these foods, fat provides $74 \%$ of energy in cheddar cheese, $78 \%$ in salami, $57 \%$ in chicken nuggets and $52 \%$ in French fries ${ }^{22}$. The observed relationship between fat intake and campylobacter infection may relate to the detergent effects of fatty foods in the small intestine. However, this does not explain the difference between groups in butter consumption (which was consumed more by cases). There may be an effect of fatty acid type, with dietary $n-3$ fatty acids (found particularly in cold-water fish) having been shown to modify gut immunological function $^{23}$.

At a more general level, fat is known to play an important role in the growth and development of young children, primarily as an energy source. It is also essential for proper functioning of the gastrointestinal system. Indeed, a history of low fat intake in infants and pre-school children may lead to prolonged diarrhoea ${ }^{24}$. In Australia, it is recommended that fat should comprise approximately
$50 \%$ of energy intake in early infancy, with a gradual lowering to $40 \%$ by 5 years of age $\mathrm{e}^{25}$. It is unlikely that fat content alone explains the current findings. Several of the 'protective' foods (besides cheese) contain other compounds that inhibit bacterial growth. For example, salami and bacon products contain preservative agents that are bactericidal $^{26}$. Consumption of chocolate was also higher by the comparison group, although not significantly (OR $0.67,95 \%$ CI $0.43-1.06, P<0.084$ ). The cocoa found in chocolate has been shown to inhibit enzyme activity of Streptococcus mutans $^{27}$, an organism implicated in dental caries.

Consumption of two foods (sweet potatoes and oat porridge) was lower by controls than by cases. This difference was close to statistical significance but is noteworthy, as there is evidence implicating sweet potato consumption in gastroenteritis. In regions of Papua New Guinea, high sweet potato intake is associated with a necrotising enteritis (pig bel) ${ }^{28}$. In this syndrome it is thought that the presence of a trypsin inhibitor in sweet potatoes coupled with infection by Clostridium perfringens (together with other complex environmental factors) are involved. It does not appear to be related to starch per se, which would be consistent with current findings as neither potatoes nor other cereal starchy foods were related to risk and consumption of hot French fries (potato chips) was higher in the controls. We have no explanation for the differences in oat porridge consumption between cases and controls.

The general lack of association of previously postulated risk factors for campylobacter infection suggests either that other influences, such as host dietary practices, are in operation in this study population or/and that our study was under-powered with regard to these variables. One of these risk factors, namely the keeping of live chickens, was found to be higher in our cases and is quite consistent with expectations, although only a small number of respondents (22 of 345) indicated ownership. This may indicate that the study was, in fact, powerful enough to detect such factors in these children.

The relationship observed between risk of campylobacter infection in children with a familial colon cancer risk is somewhat unexpected. However, a campylobacterlike organism, which may be causative, has been noted in colonic adenocarcinoma in rats ${ }^{29}$.

Clearly the present findings require replication and further examination. In particular, the relationship between the reported frequency of consumption of foods versus the amount consumed requires thorough examination. We have no means of discriminating between these two variables in this study. Previous studies $^{20}$ have focused on foods largely as potential vectors of infection. The current data suggest that foods act in a host-specific manner to alter individual susceptibility, and add another dimension to quantitative microbial-risk analysis. 


\section{Acknowledgements}

We wish to thank the Drs A McOrist, P Desmarchelier and P Ryan for useful discussion and Ms L Griffith for advice on data management. We are grateful to the participating parents, guardians and caregivers and their children for their unselfish co-operation in the conduct of the survey. The financial support of the University of Adelaide-CSIRO Health Sciences and Nutrition Trust is gratefully acknowledged.

\section{References}

1 Gracey M. Nutritional effects and management of diarrhea in infancy. Acta Paediatrica (Supplement) 1999; 88: 110-2.

2 Cohen ML. Changing patterns of infectious disease. Nature 2000; 406: 762-7.

3 Stafford R, Tenkate T, McCall B. A five year review of Campylobacter infection in Queensland. Communicable Diseases Intelligence 1996; 20: 478-82.

4 Brieseman MA. A further study of the epidemiology of Campylobacter jejeuni infections. New Zealand Medical Journal 1990; 103: 207-9.

5 Tauxe RV, Hargrett-Bean N, Patton CM, Wachsmut IK. Campylobacter isolates in the United States 1982-1986. Morbidity and Mortality Weekly Report 1988; 37(SS-2): 1-13.

6 Gibson GR, Roberfroid MB. Dietary modulation of the human colonic microbiota: introducing the concept of prebiotics. Journal of Nutrition 1995; 125: 1401-12.

7 Topping DL, Clifton PM. Short chain fatty acids and human colonic function: roles of resistant starch and non-starch polysaccharides. Physiological Reviews 2001; 81: 1031-64.

8 Bird AR, Brown IL, Topping DL. Starches, resistant starches, the gut microflora and human health. Current Issues in Intestinal Microbiology 2000; 1: 25-37.

9 Ramakrishna BS, Venkataraman S, Srinivasan S, Dash P, Young GP, Binder HJ. Amylase-resistant starch plus oral rehydration solution for cholera. New England Journal of Medicine 2000; 342: 308-13.

10 Rabbani GH, Teka T, Zaman B, Majid N, Khatun M, Fuchs GJ. Clinical studies in persistent diarrhea: dietary management with green banana or pectin in Bangladeshi children. Gastroenterology 2001; 121: 554-60.

11 Hampson DJ, Robertson ID, La T, Oxberry SL, Pethick DW. Influences of diet and vaccination on colonisation of pigs by the intestinal spirochaete Brachyspira (Serpulina) pilosicoli. Veterinary Microbiology 2000; 73: 75-84.

12 Baghurst PA, Baghurst KI, Record SJ. Dietary fibre, nonstarch polysaccharides and resistant starch - a review. Food Australia 1996; 48(Suppl.): S3-35.

13 Cassidy A, Bingham SA, Cummings JH. Starch intake and colorectal cancer risk: an international comparison. British Journal of Cancer 1994; 69: 937-42.
14 Wilson DH, Starr GJ, Taylor AW, Del Grande E. Random digit dialling and Electronic White Pages samples compared: demographic profiles and health estimates. Australian and New Zealand Journal of Public Health 1999; 23: 627-33.

15 Baghurst KI, Crawford DA, Worsley A, Record SJ. The Victorian Nutrition Survey - intakes and sources of dietary fats and cholesterol in the Victorian population. Medical Journal of Australia 1988; 149: 12-20.

16 Australian Bureau of Statistics. National Nutrition Survey 1995. Canberra: Australian Government Printing Service, 1997.

17 Stata Corp. Stata Statistical Software, Release 7. College Station, TX: Stata Corp., 2000.

18 Department of Human Services. Notifiable Disease in South Australia. Annual Summary 1996. Adelaide: Department of Human Services, 1997.

19 Anon. Topics in minority health: Yersinia entercolitica infections during the holidays in black families - Georgia. Morbidity and Mortality Weekly Report 1990; 39: 819-20.

20 Schorr D, Schmid H, Rieder HL, Baumgartner A, Vorkauf H, Burnens A. Risk factors for Campylobacter enteritis in Switzerland. Zentralblatt für Hygiene und Umweltmedizin 1994; 196: 327-37.

21 Bachmann HP, Spahr U. The fate of potentially pathogenic bacteria in Swiss hard and semihard cheeses made from raw milk. Journal of Dairy Science 1995; 78: 476-83.

22 English R, Lewis J. Food for Health. A Guide to Good Nutrition with Nutrient Values for 650 Australian Foods. Canberra: National Food Authority, Australian Government Publishing Service, 1991.

23 Kuratko CN, Becker SA. Dietary lipids alter fatty acid composition and $\mathrm{PGE}_{2}$ production in colonic lymphocytes. Nutrition and Cancer 1998; 31: 56-61.

24 Cohen SA, Hendricks K, Mathis R, Laramee S, Walker WA. Chronic non-specific diarrhea - complication of dietary fat restriction. Pediatrics 1979; 64: 402-7.

25 National Health and Medical Research Council. Dietary Guidelines for Children and Adolescents. Canberra: Commonwealth of Australia, 1995.

26 Gould GW. Methods for preservation and extension of shelf life. International Journal of Food Microbiology 1996; 33: 51-64.

27 Kashket S, Paolino VJ, Lewis DA, van Houte J. In-vitro inhibition of glucosyltransferase from the dental plaque bacterium Streptococcus mutans by common beverages and food extracts. Archives of Oral Biology 1985; 30: 821-6.

28 Murrell WG, Roth L, Egerton J, Samela J, Walker PD. Pig-bel: enteritis necroticans. A study in diagnosis and management. Lancet 1966; i: 217-22.

29 Vandenberghe J, Verheyen A, Lauwers S, Geboes K. Spontaneous adenocarcinoma of the ascending colon in Wistar rats: the intracytoplasmic presence of a Campylobacter-like bacterium. Journal of Comparative Pathology 1985; 95: 45-55. 